\title{
TEMA 5-2014: GÁNGER DE OVARIO DURANTE EL EMBARAZO
}

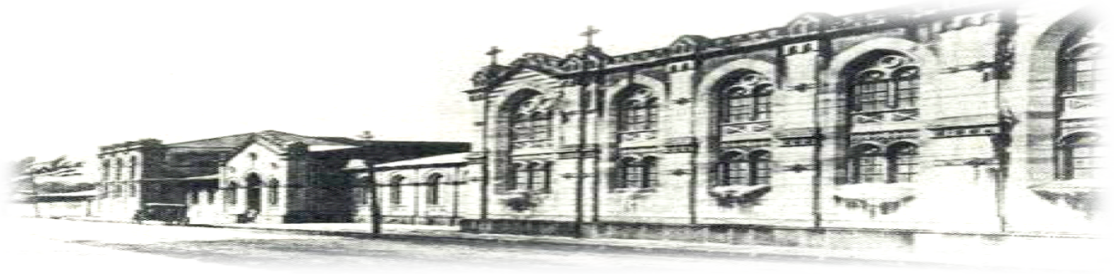

Hospital San quan de Dias. San Jasé, Casta Rica. Fundada en 1845

Recibido: $\quad 26 / 01 / 2014$

Aceptado: $\quad 26 / 06 / 2014$

Manuel Arce von Herold
${ }^{1}$
Kenneth Loáiciga Vega

${ }^{1}$ Médico general. Correo electrónico: manuelarcevonherold@gmail.com

${ }^{2}$ Médico especialista en Ginecología, Obstetricia y Ginecología Oncológica. Asistente del Servicio de Ginecología del Hospital San Juan de Dios.

\section{RESUMEN}

El cáncer durante el embarazo implica una difícil situación en la que hay que velar por dos vidas. Anteriormente el tratamiento oncológico se consideraba incompatible con el desarrollo normal del feto y se recurría a tratamientos tardíos o terminación del embarazo. Con los años, en la literatura se ha acumulado la experiencia de pequeños reportes de casos y series que han formado una imagen más clara sobre el escenario del cáncer en el embarazo. En el cáncer de ovario ahora es posible ofrecer un tratamiento adecuado a la madre sin poner al feto bajo un riesgo serio.

\section{PALABRAS CLAVE}

Cáncer de ovario. Embarazo.

\section{ABSTRACT}

Cancer during pregnancy involves a difficult situation in which two lives must be taken into account. Previously, oncological treatment was considered incompatible with normal fetal development and led to late treatment or the termination of pregnancy. With years, experience of small case reports and case series has increased in literature, which has created a clearer image of cancer during pregnancy. Regarding ovarian cancer, it is now possible to offer adequate treatment to the mother without placing the fetus under serious risk.

\section{KEY WORDS}

Ovarian cancer. Pregnancy. 


\section{INTRODUCCIÓN}

Las neoplasias malignas son diagnosticadas en aproximadamente 1 de cada 1.000 embarazos, de las cuales entre un 2 y un $10 \%$ corresponden a cáncer de ovario, es decir, 1 de cada 10.000 a 1 de cada 50.000 embarazos, siendo ésta la segunda malignidad ginecológica más frecuente, después del cáncer de cérvix ${ }^{(1-4)}$. Las masas anexiales se presentan entre un 2,3 y un $4,1 \%$ de las gestaciones, siendo los quistes dermoides y los cistadenomas las variantes histológicas más frecuentes $(28-35 \%$ y $16-24 \%$, respectivamente) (5-7). La mayoría de las masas anexiales en el embarazo son fisiológicas y revierten espontáneamente durante la gestación ${ }^{(7)}$. Sin embargo, de un 2 a un 5\% son malignas. A pesar de esto, la literatura apunta a que el embarazo no empeora el pronóstico del cáncer de ovario ${ }^{(8,9)}$.

\section{DISCUSIÓN}

\section{Diagnóstico}

Algunos de los síntomas típicos del cáncer de ovario pueden confundirse con otros propios del embarazo, tales como la distensión abdominal, mayor frecuencia urinaria o una sensación de fácil llenura. Un $14.2 \%$ de las mujeres grávidas con una masa anexial presenta torsión de la misma, lo que lleva a su diagnóstico. Entre un 65 $y$ un $80 \%$ de las masas se encuentran en pacientes asintomáticas ${ }^{(7)}$.

El diagnóstico de una masa se realiza principalmente por hallazgos ultrasonográficos, ya que la $\alpha$-feto proteína, la gonadotrofina coriónica humana, la inhibina A y el CA-125 fluctúan durante el embarazo y son menos específicos $^{(4,6)}$. El CA-125 puede elevarse durante el primer trimestre de un embarazo normal hasta $65 \mathrm{U} / \mathrm{mL}$, para después revertir a valores menores a $35 \mathrm{UI} / \mathrm{ml}$ a las 15 semanas de gestación, por lo que valores mayores a éstos, en el contexto de una masa anexial pueden ser considerados al tomar una decisión sobre la misma y se pueden utilizar en el seguimiento, aunque su utilidad sigue siendo menor que en casos de mujeres no embarazadas ${ }^{(8)}$.

La mayoría de las masas anexiales malignas se detectan en estadios tempranos, de manera incidental, en controles prenatales de pacientes asintomáticas durante la primera mitad del embarazo $^{(1,7)}$. A diferencia de la población de mujeres no embarazadas, en las que la mayoría de malignidades presentan estadiajes avanzados, en el contexto de embarazo hasta un $80 \%$ de los casos se detectan en un estadio I y menos del $15 \%$ en estadios III o IV $^{(7)}$

Los hallazgos ultrasonográficos más comúnmente asociados a una masa anexial maligna son la presencia de áreas sólidas, masas multiloculadas, líquido libre, superficies irregulares, bilateralidad, septos internos gruesos y proyecciones papilares ${ }^{(8)}$. En ciertas situaciones, se podría recurrir a la resonancia magnética para diferenciar entre masas anexiales y leiomiomas uterinos y definir mejor la morfología de la masa.

Se recomienda evitar estudios con gadolinio como medio de contraste, por los posibles efectos fetales a largo plazo que este medio pueda producir. Aunque actualmente la tomografía computarizada es comúnmente empleada al valorar el cáncer de ovario, su uso en un contexto de embarazo es muy limitado por los efectos que conlleva la radiación ionizante durante la organogénesis y el desarrollo del feto $^{(1)}$.

Para las masas sospechosas de ser malignas, el diagnóstico definitivo se realiza con la histopatología tras la resección quirúrgica.

\section{Manejo}

El primer paso en el manejo de las masas anexiales que persisten en el embarazo es determinar su potencial malignidad. Si estas masas son asintomáticas, se debe realizar cirugía solo si son sospechosas u obviamente malignas.

En aquellas pacientes con masas sospechosas, se realiza la cirugía tanto para establecer un diagnóstico como para determinar el grado histopatológico y comenzar una terapia inicial. A diferencia de la epidemiología general del cáncer de ovario, en las mujeres embarazadas los tumores son principalmente no epiteliales o de bajo potencial de malignidad; $48 \%$ son tumores limítrofes, $25 \%$ son tumores de célula germinal y los estromales del cordón sexual son menos del $5,5 \%$. Mientras que en las no embarazadas los tumores epiteliales son el grupo predominante (hasta 90\%), durante el embarazo representan solamente un $21 \%$, aunque esta cifra 
posiblemente aumentará con el incremento de mujeres que posponen sus planes de maternidad a edades más tardías ${ }^{(7,8,10)}$.

El manejo quirúrgico debe ser valorado en una ventana en que no sea demasiado temprano, lo cual podría provocar un aborto o pérdida de la función lútea, ni demasiado tardío, cuando se podría provocar una labor de parto prematura, o que el cáncer de ovario ya hubiese avanzado en estadiaje o que se corra mayor riesgo de torsión, ruptura o sangrado. Se prefiere realizar una laparotomía sobre la línea media, en el segundo trimestre, con manipulación mínima del útero grávido $^{(1)}$. Marret y colaboradores recomiendan realizar cirugía en masas que persistan durante el segundo trimestre, sean $>5-10 \mathrm{~cm}$ en diámetro o tengan características ultrasonográficas sólidas o solido-quísticas $^{(4)}$. El abordaje laparoscópico se puede realizar en masas anexiales en las que se sospecha malignidad antes de las semanas 16 a 18; si tienen un tamaño $<8 \mathrm{~cm}$, no se sospecha que sea un cáncer de estadio avanzado y se pueda remover la masa intacta y completa ${ }^{(8)}$. No se debe utilizar una aguja Verres, para evitar una posible perforación uterina ${ }^{(1)}$.

En cuanto a la quimioterapia durante el embarazo, los riesgos varían según el agente y la edad gestacional. En las primeras cuatro semanas existe un efecto de "todo o nada", en el cual el embarazo termina en un aborto o no se producen efectos secundarios del todo ${ }^{(11,12)}$. En el periodo de organogénesis, entre las 4 y 12 semanas, es cuando existe mayor riesgo de teratogenicidad, sobre todo con el uso de múltiples medicamentos.

Los principales efectos adversos en el segundo y tercer trimestre son restricción de crecimiento intrauterino, prematuridad, bajo peso al nacer y toxicidades similares a las que está expuesta la madre (ototoxicidad, pérdida de cabello, mielosupresión $)^{(13)}$. El uso de carboplatino ha sido presentado en reportes aislados y pequeñas series en las que se usó después del primer trimestre sin complicaciones maternofetales ${ }^{(8,14)}$.

Los taxanos tienen aún menos experiencia, pero aunque algunos estudios no reportaron efectos adversos para el embarazo con su uso en el segundo o tercer trimestre ${ }^{(15,16)}$, otros han reportado prematuridad con síndrome de insuficiencia respiratoria del infante y estenosis pilórica con su uso en otras malignidades ${ }^{(17)}$.
El régimen quimioterapéutico de elección para el cáncer de ovario epitelial es paclitaxel con carboplatino y para el de célula germinal es bleomicina con etopósido y cisplatino. Todos estos agentes son categoría D. De aplicarse durante el embarazo, la última quimioterapia debe darse 4 semanas antes de la fecha esperada de parto, para evitar que coincida con mielosupresión o aplasia y se den posibles complicaciones tanto en la madre como en el neonato, incluyendo sangrado, sepsis y muerte ${ }^{(4)}$.

Aunque la fisiología del embarazo conlleva un distinto aclaramiento de los fármacos, ante la ausencia de estudios se emplean las mismas dosis por peso y talla en mujeres embarazadas que en las que no lo están ${ }^{(1)}$.

Los tumores limítrofes (borderline), de célula germinal y los estromales de cordones sexuales suelen diagnosticarse en estadio I. Aproximadamente un $75 \%$ de los de célula germinal en el embarazo son disgerminomas ${ }^{(18)}$, mientras que los estromales de cordón sexual son en un $50 \%$ tumores de las células de la granulosa y un tercio tumores de células de Sertoli o Leydig $^{(19)}$. Durante el embarazo, a estas distintas masas se les puede realizar una laparotomía en línea media con salpingo-ooforectomía unilateral, omentectomía, citología peritoneal y biopsias de los distintos órganos y tejidos. En estadios más avanzados se busca remover ambos anexos durante el embarazo y completar la cirugía después del parto ${ }^{(1)}$. Para el cáncer de ovario de célula germinal existe muy poca evidencia para dar una clara recomendación basada en sobrevida materna sobre si comenzar un esquema de quimioterapia durante el embarazo o después del parto.

Para el carcinoma epitelial de ovario el tratamiento varía según el estadio y la histología. Para estadios IA y IB, grados 1 y 2 y de histología favorable (seroso, mucinoso o endometrioide) se puede tener un manejo expectante y realizar un estadiaje quirúrgico completo después de un parto electivo a las 34 semanas. Un abordaje que preserve la fertilidad se recomendaría solo en mujeres con estadios IA, grado 1 y 2 que tengan un estadiaje quirúrgico completo y adecuado, sin histología de células claras y que desean procrear aún ${ }^{(8,20)}$. Los estadios IA y IB con histología desfavorable (células claras) o grado 3 , y los estadios IC y II presentan entre un 30 y $50 \%$ de recurrencias en 5 
años, por lo que no son buenas candidatas para terapias que preservan la fertilidad. Su sobrevida general y la sobrevida sin progresión de la enfermedad se extienden con el uso de seis ciclos de carboplatino y paclitaxel después de un estadiaje quirúrgico, lo cual presenta tres escenarios: en el más recomendado, este abordaje se lleva a cabo después de una cesárea a las 34 semanas, pero las opciones son realizarlo durante el embarazo (estadiaje completo y adyuvancia) o comenzar un esquema de tres o cuatro ciclos de quimioterapia neoadyuvante a partir de las 14 semanas y después del parto hacer el estadiaje quirúrgico completo ${ }^{(8)}$.

En el cáncer de ovario epitelial de estadio avanzado (III o IV), más del $70 \%$ de las mujeres presentan una recurrencia en dos años y $70 \%$ fallecen en los primeros cinco años del diagnóstico, por lo que el tratamiento inmediato es lo recomendado. Durante el embarazo se han descrito distintos abordajes, tales como resección completa con terminación del embarazo, cirugía durante el embarazo con quimioterapia postparto, y quimioterapia en el embarazo seguido de cirugía final postparto $^{(1)}$. En algunas pacientes se puede realizar una citorreducción completa antes de las 12 semanas. De no ser posible, usualmente se recomienda una terminación del embarazo, ante la incertidumbre de la quimioterapia como teratogénico en el primer trimestre. $\mathrm{Si}$ el diagnóstico se hace en el segundo trimestre se puede continuar con el embarazo y comenzar un régimen neoadyuvante o de citorreducción sin comprometer la gesta, mientras que el diagnóstico en el tercer trimestre indicaría una cesárea con estadiaje completo y adyuvancia ${ }^{(8)}$.

\section{CONCLUSIONES Y RECOMENDACIONES}

El manejo del cáncer de ovario durante el embarazo requiere un trabajo en equipo por parte de ginecólogos oncólogos, patólogos, anestesiólogos, obstetras, oncólogos médicos, neonatólogos y enfermeros, entre otros especialistas con experiencia en tratamiento integral del cáncer.

Los escenarios que se discuten al lado de la paciente y su familia permiten que hoy se tomen decisiones en beneficio tanto de la madre como de su bebé. La experiencia ha permitido entender mejor los posibles riesgos y así comprender las distintas opciones terapéuticas que se plantean hoy en día

\section{REFERENCIAS BIBLIOGRÁFICAS}

1. Amant F Brepoels L Halaska MJ Gziri MM Calsteren KV. Gynaecologic cancer complicating pregnancy: an overview. Best Pract Res Clin Obstet Gynaecol. 2010;24(1):61-79.

2. Oehler MK Wain GV Brand A. Gynaecological malignancies in pregnancy: a review. Aust N Z J Obstet Gynaecol. 2003;43(6):414-420.

3. Whitecar MP Turner S Higby MK. $A d-$ nexal masses in pregnancy: a review of 130 cases undergoing surgical management. Am J Obstet Gynecol. 1999; 181(1):19-24.

4. Marret H Lhommé C Lecuru F et al. Guidelines for the management of ovarian cancer during pregnancy. Eur J Obstet Gynecol Reprod Biol. 2010;149(1): 18-21.

5. Bernhard LM Klebba PK Gray DL Mutch DG. Predictors of persistence of adnexal masses in pregnancy. Obstet Gynecol. 1999;93(4):585-589.

6. Giuntoli RL Vang RS Bristow RE. Evaluation and management of adnexal masses during pregnancy. Clin Obstet Gynecol. 2006;49(3):492-505.

7. Aggarwal P Kehoe S. Ovarian tumours in pregnancy: a literature review. Eur J Obstet Gynecol Reprod Biol. 2011;155 (2):119-124.

8. Minig L Otaño L Diaz-Padilla I Alvarez Gallego R Patrono MG Valero de Bernabé J. Therapeutic management of epithelial ovarian cancer during pregnancy. Clin Transl Oncol. 2013;15(4):259264.

9. Ferrandina G Distefano M Testa A De Vincenzo R Scambia G. Management of an advanced ovarian cancer at 15 weeks of gestation: case report and literature review. Gynecol Oncol. 2005;97 (2):693-696.

10. He SY Shen HW Xu L Li XL Yao SZ. Successful management of mucinous ovarian cancer by conservative surgery in week 6 of pregnancy: case report and literature review. Arch Gynecol Obstet. 2012;286(4):989-993. 
11. Cardonick E Iacobucci A. Use of chemotherapy during human pregnancy. Lancet Oncol. 2004;5(5):283-291.

12. Leslie KK Koil C Rayburn WF. Chemotherapeutic drugs in pregnancy. Obstet Gynecol Clin North Am. 2005;32(4): 627-640.

13. Van Calsteren K Heyns L De Smet F et al. Cancer during pregnancy: an analysis of 215 patients emphasizing the obstetrical and the neonatal outcomes. $\mathrm{J}$ Clin Oncol. 2010;28(4):683-689.

14. Mir O Berveiller P Ropert S Goffinet $\mathrm{F}$ Goldwasser F. Use of platinum derivatives during pregnancy. Cancer. 2008; 113(11):3069-3074.

15. Cardonick E Bhat A Gilmandyar D Somer R. Maternal and fetal outcomes of taxane chemotherapy in breast and ovarian cancer during pregnancy: case series and review of the literature. Ann Oncol. 2012;23(12):3016-3023.

16. Zagouri F Sergentanis TN Chrysikos D Filipits M Bartsch R. Taxanes for ovarian cancer during pregnancy: a systematic review. Oncology. 2012;83(4):234238.

17. Mir O Berveiller $\mathrm{P}$ Goffinet $\mathrm{F}$ et al. Taxanes for breast cancer during pregnancy: a systematic review. Ann Oncol. 2010;21(2):425-426.

18. Bakri YN Ezzat A Akhtar Dohami Zahrani. Malignant germ cell tumors of the ovary. Pregnancy considerations. Eur J Obstet Gynecol Reprod Biol. 2000;90(1):87-91.

19. Young RH Dudley AG Scully RE. Granulosa cell, Sertoli-Leydig cell, and unclassified sex cord-stromal tumors associated with pregnancy: a clinicopathological analysis of thirty-six cases. Gynecol Oncol. 1984;18(2):181-205.

20. Amant F Van Calsteren K Halaska MJ et al. Gynecologic cancers in pregnancy: guidelines of an international consensus meeting. Int J Gynecol Cancer. 2009;19(Supp11):S1-S12.

\section{DECLARACIÓN DE CONFLICTO DE IN- TERESES}

Los autores declaran que no existen conflictos de intereses. 